\title{
New insights into the pathogenesis of asthma
}

\author{
Jack A. Elias, Chun Geun Lee, Tao Zheng, Bing Ma, Robert J. Homer, and Zhou Zhu \\ Yale University School of Medicine, New Haven, Connecticut, USA \\ J. Clin. Invest. 111:291-297 (2003). doi:10.1172/JCI200317748.
}

\section{Historical perspective}

Asthma is a disease whose ability to cause episodic symptomatology has been appreciated since antiquity. Although the fine points of the definition can be debated, it is reasonable to think of asthma as a pulmonary disorder characterized by the generalized reversible obstruction of airflow and to define reversibility as a greater than $12 \%$ increase in the patient's forced expiratory volume in 1 second $\left(\mathrm{FEV}_{1}\right)$ that occurs either spontaneously or with therapy. Airway hyperresponsiveness, an exaggerated bronchospastic response to nonspecific agents such as methacholine and histamine or specific antigens, is the physiologic cornerstone of this disorder. A diagnosis of asthma is established based on a history of recurrent wheeze, cough, or shortness of breath, reversible airway obstruction demonstrated by pulmonary-function testing, and, in cases where questions exist, a methacholine challenge demonstrating airway hyperresponsiveness. It has long been assumed that patients with asthma experience intermittent attacks and have relatively normal lung function during intervening periods. More recent studies have demonstrated that asthma can cause progressive lung impairment and, in some patients, eventuate in partially reversible or irreversible airway obstruction.

Any discussion of asthma must take into account the recent increase in its prevalence. Since approximately 1980, the frequency of this disorder has almost doubled. As a result of this "epidemic," asthma now affects approximately $8-10 \%$ of the population in the US, is the leading cause of hospitalization among children less than 15 years of age, and costs society billions of dollars annually. This increase in prevalence is not simply due to diagnostic transference or increased diagnostic awareness, since asthma mortality rates have also increased during this interval.

The Science in Medicine series is supported in part by a generous grant from the Doris Duke Charitable Foundation.

Address correspondence to: Jack A. Elias, Yale University School of Medicine, Section of Pulmonary and Critical Care Medicine, 333 Cedar Street/105 LCI, PO Box 208057, New Haven, Connecticut 06520-8057, USA. Phone: (203) 785-4163; Fax: (203) 785-3826; E-mail: Jack.Elias@yale.edu.

Conflict of interest: The authors have declared that no conflict of interest exists.

Nonstandard abbreviations used: $\mathrm{T}$ regulatory [cell] $(\mathrm{Tr})$; signal transducer and activator of transcription (STAT); chemokine receptor 2 (CCR2); histone deacetylase (HDAC).
An aerosol antigen challenge of an appropriately sensitized asthmatic patient can induce two types of airway responses. The early response is an acute bronchospastic event that occurs 15-30 minutes after exposure and resolves over time. The late-phase response peaks 4-6 hours after exposure and can cause prolonged symptomatology. Over the years, a variety of concepts of pathogenesis have been put forth in an attempt to explain one or both of these responses (Table 1). Early investigators postulated that there was an intrinsic airway smooth muscle abnormality at the root of the asthmatic diathesis. However, many studies with airway myocytes in culture have not corroborated this contention. This was followed by the contention that asthma is an autonomic dysfunction syndrome characterized by excess cholinergic and/or tachykinin pathway activity. This was never proven or disproven. Instead, IgE-mediated mast cell and/or basophil degranulation with the release of leukotrienes, histamine, prostaglandins, tryptase, cytokines (such as IL-4 and IL-5), and other mediators was appreciated to be a key event in the acute response. The prominent eosinophil-, macrophage-, and lymphocyte-rich inflammatory response in the airways of patients with asthma (Figure 1) and the efficacy of steroids in the majority of patients with asthma then led to the present-day concept that asthma is a chronic inflammatory disorder of the airway and that $\mathrm{T}$ cells are pivotal initiators and regulators of this response. Structural alterations including airway wall thickening, fibrosis in the lamina reticularis and adventitia of the airway, mucus metaplasia, myocyte hypertrophy and hyperplasia, and neovascularization are all readily appreciated in the asthmatic airway (Figure 1). This led to the hypothesis that the inflammatory response in the asthmatic airway causes these remodeling events, and to the belief that these events contribute to disease pathogenesis. Studies using new immunologic and molecular approaches have provided impressive insights into the nature of this inflammatory response and the relationship between this response and the remodeling and physiologic alterations characteristic of the disorder.

\section{The Th1/Th2 paradigm}

It has been known for over 50 years that people tend to mount antibody- or cell-mediated immune responses to specific antigens. A major advance in our knowledge of the mechanisms responsible for these divergent effects was achieved when it was discovered, initially in studies in mice, that the type of response that is seen is 


\section{Table 1}

Evolving concepts of asthma pathogenesis

1. A primary abnormality of airway-myocyte hyperresponsiveness

2. Autonomic dysfunction with exaggerated activity of cholinergic or tachykinin pathways

3. IgE-mediated mast cell/basophil degranulation

4. Complex T lymphocyte-mediated airway inflammation

5. Airway remodeling

influenced greatly by the type of T cells that accumulate at the site of local antigen deposition. In the mouse, a number of functionally distinct $\mathrm{CD} 4^{+} \mathrm{T}$ cells have been defined based on the profile of cytokines that they elaborate. Although the differentiation is not as clear in humans, similarly differentiated cells in humans have been described. Th 1 and Th 2 cells have been the topic of the most intense study, with the former elaborating IFN- $\gamma$, IL- 2 , and lymphotoxin, and the latter elaborating IL-4, IL-5, IL-9, IL-13, and IL-10. Significantly, less is known about $T$ regulatory $(\mathrm{Tr})$ cells and Th 3 cells, which produce IL-10 and TGF- $\beta_{1}$, respectively $(1,2)$. As shown in Figure 2, Th1 and Th 2 cells are formed from a common naive precursor $\mathrm{T}$ cell and differentiate into polarized populations based on signals from the local microenvironment. In the presence of CD $8 \alpha^{+}$DCs and/or IL-12, IL-18, or IFN- $\gamma$, they differentiate into Th 1 cells. This evolution is mediated by a mechanism that is dependent on signal transducer and activator of transcription-1 (STAT-1) and the T-bet transcription factor $(1,3)$. In the presence of $C D 8 \alpha$ DCs and/or IL-4 (which can come from IgE-activated mast cells or DCs), Th2 cells are formed. This is a complex process that involves STAT-6-mediated signal transduction and the activation of a variety of transcription factors, including GATA-3, nuclear factor of activated $\mathrm{T}$ cells-c $\left(\mathrm{NFAT}_{\mathrm{c}}\right)$, and $\mathrm{c}$-maf $(2,4)$. Interestingly, Th1/Th2 counter-regulation has also been described, with each cell population able to inhibit and/or regulate the development and/or phenotype induced by the other. Th1 polarized responses play a key role in macrophage activation in delayed-type hypersensitivity reactions. They are a key feature in the pathogenesis of diseases like rheumatoid arthritis, sarcoidosis, and tuberculosis. In contrast, Th2-dominant responses stimulate antibody-mediated responses, activate mast cells, and elicit tissue eosinophilia. They play a key role in allergy and antiparasite responses. They are also the predominant responses in the asthmatic airway, where elevated levels of IL-4, IL-5, IL-13, and IL-9 have been detected by a variety of investigators (5).

\section{Characterization of the chronic effector functions of Th2 cytokines}

In contrast to the vast majority of injury and repair responses in the lung and other organs, asthmatic inflammation frequently starts in childhood and persists throughout the life of the afflicted individual. In addition, physicians treating asthmatics invariably find themselves attempting to deal with manifestations of established disease, often in the setting of a disease exacerbation. Surprisingly, the model systems that have been used most frequently in studies designed to understand asthma pathogenesis have not appropriately taken these issues into account. Instead, the most commonly employed modeling systems evaluate the acute responses that are elicited in the lung after normal animals are sensitized to and then challenged with an aeroallergen. The asthma-like inflammation and physiologic dysregulation that are seen in these models are an end result of the cellular and molecular events involved in sensitization, Th2 cell development, Th2 cytokine elaboration, and the activation of Th2 cytokine effector pathways. Interventions that inhibit any of these steps can appear to have a beneficial effect on the asthma-relevant readouts that are employed. However, since it is likely that antigen sensitization, Th2 cell development, and Th2 cytokine elaboration have already occurred in patients with established disease and/or a disease exacerbation, interventions at these sites will likely be less than useful therapeutically. In contrast, interventions that regulate Th 2 cytokine effector pathways are attractive as therapies. Until recently, modeling systems that allowed the inflammatory and remodeling effects of chronically elaborated Th2 cytokines to be selectively evaluated did not exist. Overexpression-transgenic methodology has, however, powerfully addressed this issue.

The standard approaches used to generate overexpression-transgenic mice are illustrated in Figure 3. First, a DNA construct is prepared that contains the gene that the investigator wishes to express and a promoter to drive the expression of this gene in the desired organ and/or tissue (Figure 3a). If temporally regulated gene expression is desired, recent advances in transgenic methodology that involve the generation of double- and triple-transgenic animals allow the transgene to be selectively turned on or off at any time during the life of the animal $(6,7)$. When asthma-relevant questions are being asked, the Clara cell $10-\mathrm{kDa}$ protein (CC10) promoter is used to target gene expression, because it is selectively expressed by

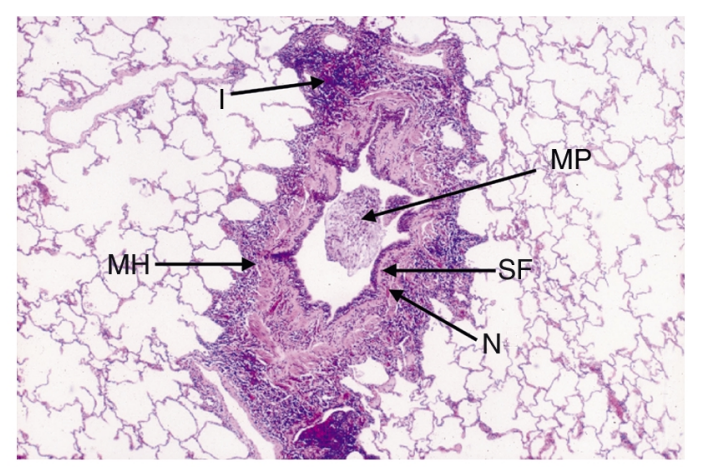

Figure 1

Inflammation and remodeling in the asthmatic airway. There is impressive inflammation (I), mucus plugging (MP), subepithelial fibrosis (SF), myocyte hypertrophy and hyperplasia $(\mathrm{MH})$, and neovascularization $(\mathrm{N})$ in this autopsy lung section from a teenage asthmatic individual. 

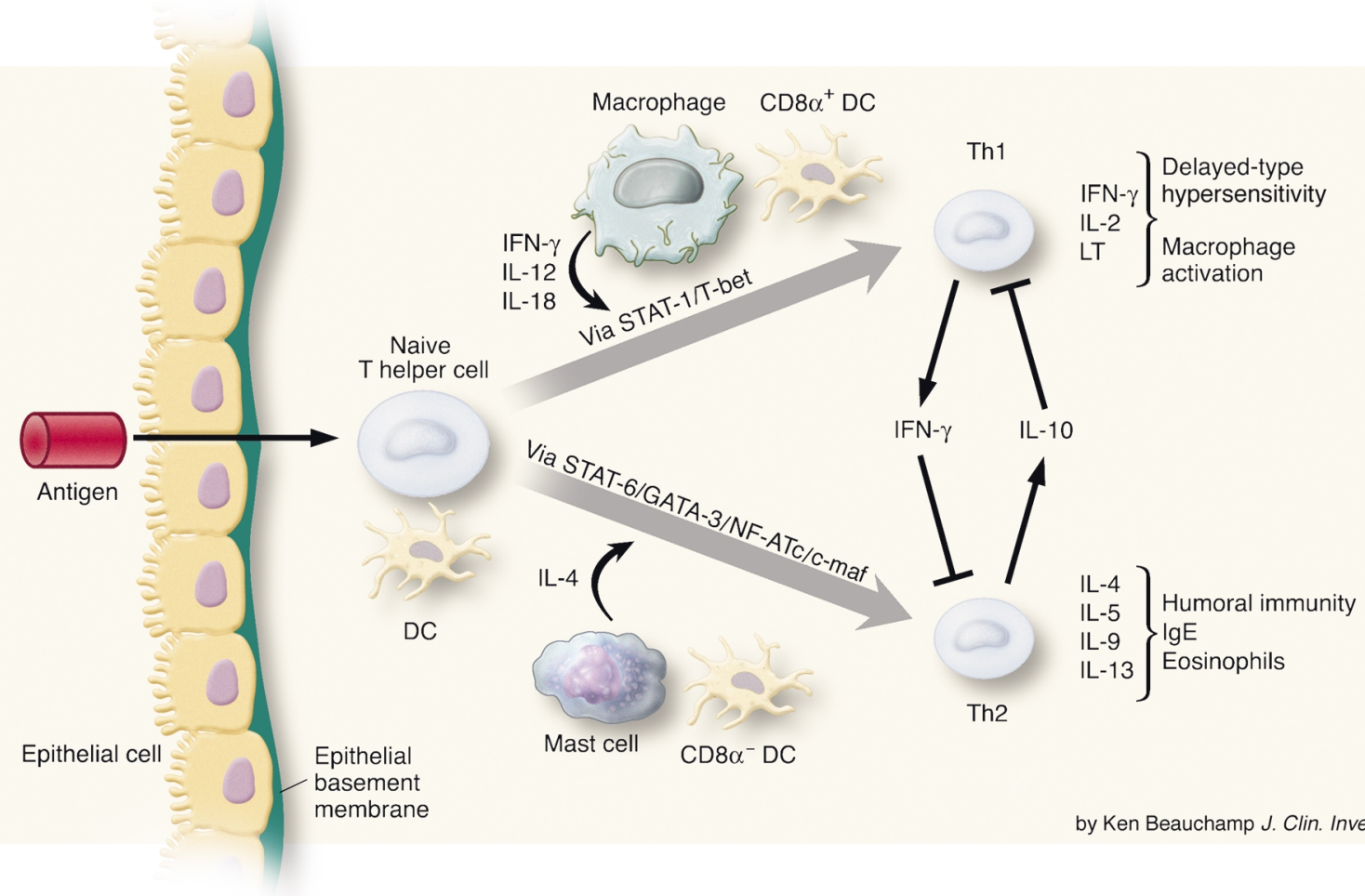

by Ken Beauchamp J. Clin. Invest.

\section{Figure 2}

Development of Th1 and Th2 lymphocytes. Antigens enter through the endobronchial tree, cross the epithelial surface, and interact with naive Th cells and DCs. As a result of signals from the surrounding microenvironment, they differentiate into Th1 cells, which produce IFN- $\gamma$, IL-2, and lymphotoxin (LT), or Th2 cells, which produce IL-4, IL-5, IL-9, IL-13, and IL-10. Polarization into Th1 cells occurs via a STAT-1- and T-bet-dependent pathway under the influence of CD $8 \alpha^{+}$DCs and macrophage-derived cytokines such as IFN- $\gamma$, IL-12, and IL-18. Differentiation into Th2 cells occurs via a pathway that involves STAT-6, GATA-3, nuclear factor of activated T cells-c (NFAT $)$, and c-maf under the influence of CD8 $\alpha^{-}$DCs and IL-4, which may come from mast cells.

the Clara cells that make up $40 \%$ of the epithelium of the murine airway. To generate transgenic mice, male and female mice are allowed to mate, and the fertilized eggs are washed out of the female's oviduct. The desired DNA construct is then directly microinjected into the pronuclei of these eggs, and the eggs are placed in the uterus of a pseudopregnant mouse. A pseudopregnant mouse is a female that has been mated with a vasectomized male. She is behaving, from a hormonal perspective, as if she is pregnant and becomes pregnant when the fertilized eggs are deposited in her uterus. She subsequently carries to term, delivering a litter of pups, some of which have the transgene randomly integrated into their genome, others of which do not (Figure $3 \mathrm{~b}$ ). Transgene-positive and -negative mice can be differentiated by extracting DNA from tail biopsies from the pups and determining whether the transgene is present by Southern or PCR analysis. Thus, an outstanding experimental system is established where one can compare the phenotypes of mice born to the same mother, on the same day, that are exposed to the same environment and differ only in the one gene that was inserted. The power of this approach can be easily appreciated in studies designed to define the effects of IL-13 in the asthmatic airway (8).
IL-13 is the product of a gene on chromosome 5 at q31, a site that has been repeatedly implicated in genetic studies looking for the genes involved in the asthmatic diathesis. It was originally discovered as an IL-4-like molecule and was presumed to have an effector profile identical to that of IL-4. It has since become clear that IL-13 and IL-4 differ in their effector properties, with IL-4 and IL-13 playing more prominent roles in the initiation and the effector phases of Th2 inflammation, respectively.

The effector functions of IL-13 were defined and clarified using overexpression-transgenic modeling systems. These studies demonstrated that IL-13 is a potent inducer of an eosinophil-, macrophage-, and lymphocyte-rich inflammatory response, airway fibrosis, mucus metaplasia, and airway hyperresponsiveness (8) (Figure 4). These studies also demonstrated that other Th2 cytokines, such as IL-9, mediate their effects in the lung via their ability to induce IL-13 (9), suggesting that IL-13 may be a final common pathway for Th2-mediated inflammatory responses. Importantly, these transgenic systems were also manipulated to define the mechanisms by which IL-13 generates these critical tissue responses. This was done using standard methods of quantitating mRNA and gene chip methodology to define the 

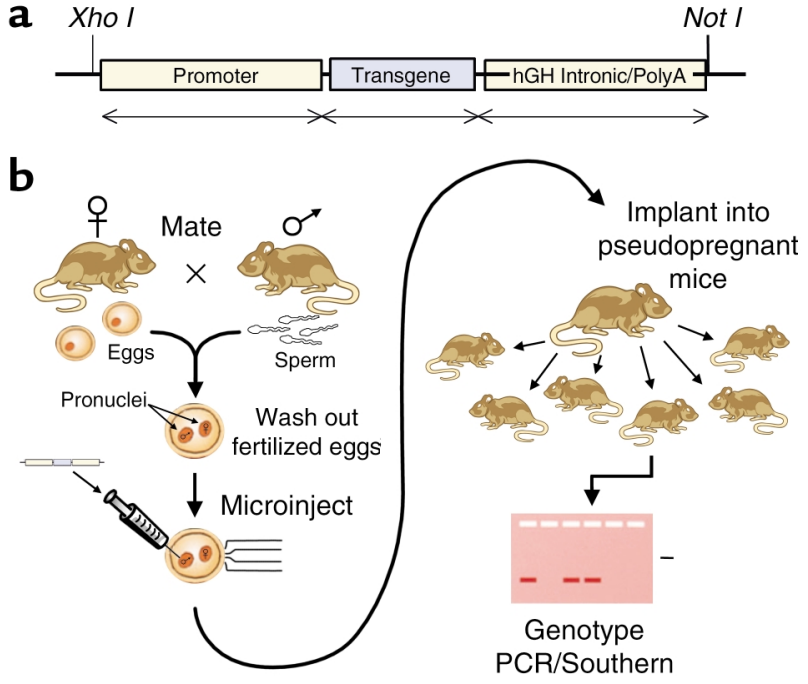

Figure 3

Generation of transgenic mice. To express a transgene in vivo, the investigator first makes a construct containing the transgene being evaluated. A typical construct is illustrated in a. It contains a promoter that targets the transgene to the desired organ, the transgene being expressed, and intronic and polyadenylation sequences that ensure the proper processing of the mRNA transcripts that are produced. The methodology for generating transgenic mice is illustrated in $\mathbf{b}$. Fertilized eggs are washed out of the oviducts of female mice. They are then microinjected under direct visualization and implanted into the uterus of pseudopregnant female mice. The genotype of the pups that are produced is evaluated in tail biopsy-derived DNA using PCR reactions or Southern blot evaluations.

genes that are regulated by IL- 13 in lungs from transgenic mice. This was followed by a variety of manipulations that characterized the contributions of specific genes to the pathogenesis of the IL-13 phenotype. One such manipulation was the use of neutralizing antibodies against the gene products in question. Another was the breeding of the IL- 13 transgenic mice with mice with null mutations of selected downstream genes, followed by characterization of the effects of the transgene in mice that were sufficient or deficient in the downstream gene in question (Figure 5 ). As can be seen in Figure 6, these studies have provided impressive insights into the mechanisms of IL-13-induced inflammation and tissue fibrosis. The inflammatory response is mediated by the ability of IL-13 to stimulate the elaboration of chemotactic cytokines called chemokines and proteolytic enzymes called matrix metalloproteinases (MMPs) $(10,11)$. These studies demonstrate that chemokine receptor 2 (CCR2), MMP-9, and MMP-12 play key roles in these responses $(10,11)$. They also demonstrate that the fibrotic response results from the ability of IL-13 to stimulate the production and activation of the fibrogenic cytokine TGF- $\beta_{1}$, and that TGF- $\beta_{1}$ is activated via an MMP-9- and plasmin-dependent pathway in this setting (12). These studies provide a road map that defines the pathways that IL-13 uses to generate tissue inflammatory and remodeling responses.
They also highlight target genes against which therapies can be directed to control selected aspects of the IL-13-induced tissue response.

\section{Contributions of new pathogenic insights to the therapy of asthma}

For decades, the overt bronchospasm and impressive benefit that patients experienced with bronchodilators led physicians and patients to rely heavily on these agents to control asthmatic symptomatology. Although steroids were also well known for their ability to control asthmatic symptoms, their side effect profile caused them to be used only in the most severe cases. The appreciation that asthma is an inflammatory disorder, the availability of effective aerosol steroid preparations, and the belief that chronic unchecked inflammation leads to airway remodeling all contributed to a change in this pattern of practice. This change is reflected in the treatment guidelines for asthma that have been promulgated in recent years by the NIH and other organizations (13). These guidelines stress the need for anti-inflammatory therapy for all but the mildest patients with infrequent symptoms. At present, steroids are the cornerstone of this anti-inflammatory intervention. However, recent advances in our understanding of asthma pathogenesis have provided insights into the mechanisms by which some of our present therapies alter airway inflammation and have provided the rationale for
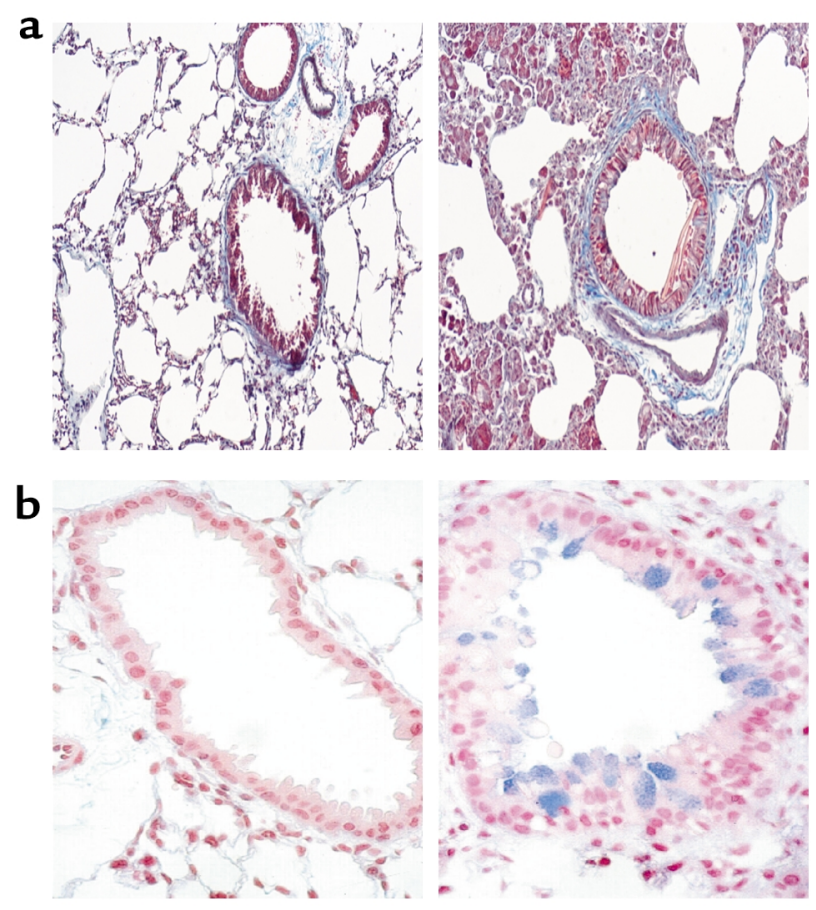

\section{Figure 4}

Demonstration of the effects of transgenic IL-13 on airway fibrosis and mucus metaplasia. (a) Trichrome stains are used to compare the amount of blue-staining collagen around airways from transgene-negative mice (left) and transgene-positive mice (right). (b) Alcian blue stains are used to demonstrate mucus accumulation in airways from transgene-negative mice (left) and transgene-positive mice (right). Mucus is blue in this evaluation. 


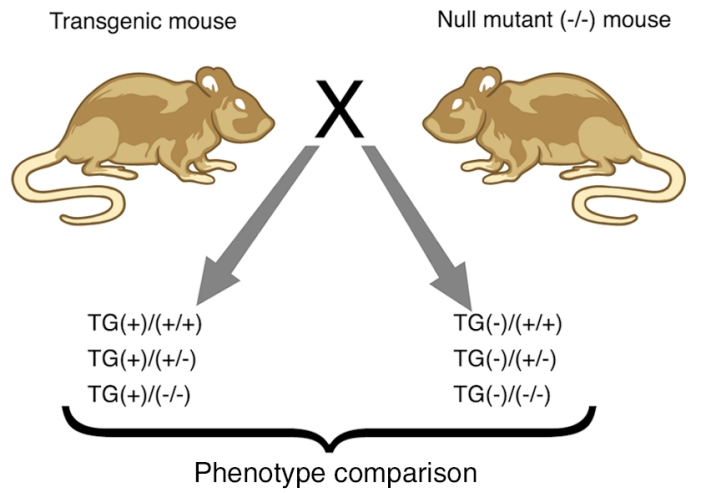

\section{Figure 5}

Use of null mutant (knockout) mice to define the pathways that transgenes use to generate disease-relevant phenotypes. In these experiments, transgenic mice with a disease-relevant phenotype (for example, fibrosis or inflammation) are mated with mice that have a null mutation of a downstream gene that is believed to play an important role in the generation of this phenotype. Transgene-positive $(\mathrm{TG}[+])$ and transgene-negative (TG[-]) mice are generated that have normal downstream genes $(+/+)$, are heterozygote knockout at the downstream gene in question $(+/-)$, or are null-mutant for the downstream gene in question $(-/-)$. The presence and intensity of the phenotypes of these mice are then compared. These comparisons allow an investigator to define the role(s) that this downstream gene plays in the generation of the pathologic response.

therapies directed against new selective inflammation-regulating targets that may have better side effect profiles than the current therapies. Lastly, our new insights raise the possibility of interventions that might actually prevent asthma in at-risk individuals. Each is reviewed below.

\section{Inflammation regulating the effects of standard therapies}

Over sixty years ago, slow-reacting substance of anaphylaxis (SRSA) was appreciated as a spasmogenic activity in lung effluent. Subsequent studies demonstrated that SRSA was mediated by leukotriene products of arachidonic acid metabolism and defined the pathways responsible for the generation of $\mathrm{LTB}_{4}$ and the cystinyl leukotrienes $\left(\mathrm{LTC}_{4}, \mathrm{LTD}_{4}\right.$, and $\left.\mathrm{LTE}_{4}\right)$. They also demonstrated the striking accumulation of cystinyl leukotrienes in biologic fluids from patients with asthma, and the bronchospasm and inflammation generating effects of these agents. As a result of these efforts, we now have cystinyl leukotriene receptor antagonists and inhibitors of 5-lipoxygenase, the enzyme that initiates the breakdown of membrane arachidonic acid. These agents have received regulatory approval for asthma therapy and are the first new therapies to be licensed for asthma in over thirty years. Their ability to improve lung function and ameliorate aspects of asthmatic inflammation is well documented. Recent studies have also demonstrated that these agents may also control tissue fibrotic responses such as that in asthmatic airway remodeling (14).

Although theophyllines have been used to treat asthma for over seventy years, their use has declined in recent decades because of their low therapeutic index and the appreciation that their anti-inflammatory effects are modest compared with those of corticosteroids. However, theophyllines can diminish airway hyperresponsiveness. In addition, the administration of low-dose theophylline to patients on steroids gives a greater improvement in asthma control than can be achieved by a doubling of the steroid dose itself. Recent studies have demonstrated that these effects may be mediated by a novel mechanism relating to chromatin remodeling. In a quiescent state, the chromatin in genes is tightly wound around core histone proteins. During gene activation, histones are acetylated, which unwinds the chromatin, allowing transcription factors and RNA polymerase II to bind and increase gene transcription $(15,16)$. This process is mediated by histone acetyl transferase (HAT). Corticosteroids inhibit this process by recruiting histone deacetylase (HDAC), which suppresses the activity of HAT. This suppression leaves the chromatin densely wound and, as a result, decreases target gene transcription. Patients with asthma have decreased levels of HDAC at base line (15). In addition, although steroids increase the levels of HDAC in asthmatic patients, those levels remain well below those induced by steroids in normal individuals (15). This suggests that therapies that increase HDAC activity will prove useful in the pharmacologic management of asthma. Interestingly, low-dose theophylline has recently been shown to augment the activation of HDAC by steroids (16). This highlights a mechanism by which low-dose theophylline can be used to augment anti-inflammatory effects of steroids in asthmatic tissues.

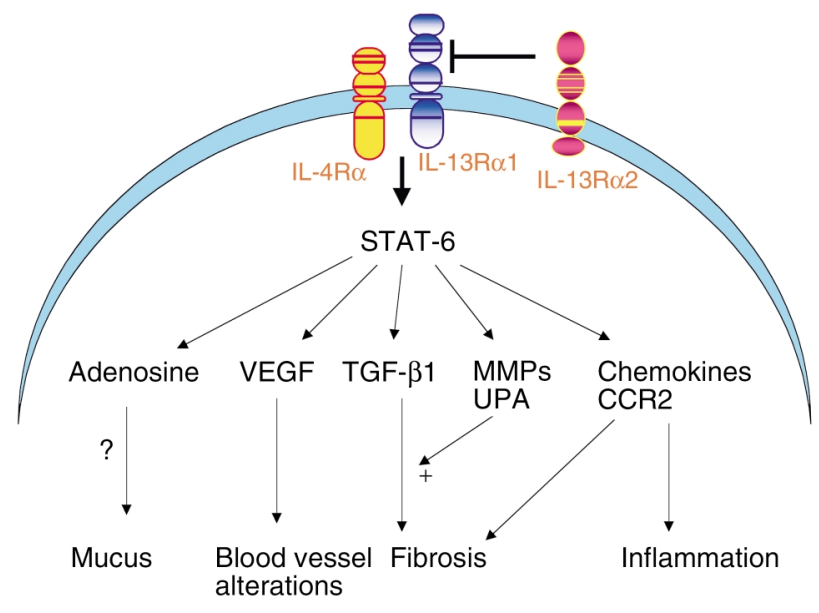

\section{Figure 6}

Mechanisms of IL-13-induced phenotype generation. IL-13 binds to the IL-13 receptor complex made up of IL-4 receptor $\alpha$ (IL-4R $\alpha$ ) and IL-13 receptor $\alpha_{1}\left(\mathrm{IL}-13 R \alpha_{1}\right)$. IL-13 also binds to IL-13R $\alpha_{2}$, which is a decoy receptor that inhibits IL-13 responses. After binding to the IL-13 receptor complex, IL-13 activates STAT- 6 signal transduction pathways. Pathways that involve chemokines, the chemokine receptor CCR2, MMPs, urinary plasminogen activator (UPA), TGF- $\beta_{1}$, VEGF, and/or adenosine are then activated, and inflammation, fibrosis, blood vessel alterations, and mucus responses are generated. Each of these pathways is a site against which therapeutic agents can be directed. 


\section{Site-specific interventions}

Advances in our understanding of the pathogenesis of asthma have opened the door to a variety of new targets against which novel therapies can be directed. It is beyond the scope of the present article to describe all of the targets that are being studied. It is, however, convenient to categorize them as interventions that alter the development of Th2 immune responses and interventions that alter the effector functions of Th2 cytokines that have been elaborated. The generation of a Th2 response requires antigen presentation by APCs such as DCs, and T cell proliferation and Th2 differentiation. These events can be inhibited by blockade of APC function (e.g., of costimulatory molecules like B7.2 or the inducible costimulator ICOS) and by blockade of $\mathrm{T}$ cell proliferation and/or differentiation (e.g., blockade of IL-4 or GATA-3, or the administration of IFN- $\gamma$ or IL-12). Examples of interventions that block Th2 effector pathways include therapies that block IL-5, IL-13, or IL-9, decrease eosinophil influx and effector function, and/or alter the production and/or effector pathways of inflammation-inducing chemokines. Treatment with a humanized antibody against IgE also alters effector pathways and has shown promise in preliminary investigations (17). Since IL-13 may be a final common pathway for Th2 cytokines (9), approaches that control IL-13 are particularly appealing (18). As illustrated in Figure 6, this includes therapies directed at the STAT- 6 signal transducer that mediates the effects of IL-13, therapies directed against the multimeric IL-13 receptor system (IL-4 receptor $\alpha$ and IL-13 receptor $\alpha_{1}$ ), and the administration of IL-13 receptor $\alpha_{2}$, a decoy receptor protein that binds IL-13 but does not transduce a signal. It also includes therapies directed against the genes that are downstream of IL-13 and mediate its effects, for example, CCR2 (11).

\section{Preventing asthma}

Our present therapeutic approach to asthma focuses exclusively on symptom amelioration. However, our knowledge of the processes that regulate Th1 and Th2 immune responses has raised the possibility of designing interventions that can prevent asthma in at-risk individuals. Many of these approaches are based on in vitro studies demonstrating that Th1 cytokines (such as IFN- $\gamma$ ) can inhibit Th2 cell development, and in vivo observations demonstrating that Th1 responses can feed back to diminish Th2 tissue responses. A variety of approaches have been proposed to accomplish this, including the administration of IFN- $\gamma$ or IFN- $\gamma$-inducing cytokines such as IL-12, and the administration of infectious agents or vaccinations that induce Th1 immunity, such as Mycobacterium bovis bacilli CalmetteGuérin (BCG). In addition, therapies have been proposed that entail the administration of oligodeoxynucleotides that contain unmethylated CPG motifs. Unmethylated CPG DNA motifs are more common in bacterial than in mammalian DNA. The administration of these agents mimics infections and induces Th1 inflammation via a Toll-like receptor 9-dependent mechanism. The ultimate utility of each of these approaches is unclear. This is due, in part, to safety concerns because it is now known that Th1 responses can contribute to the initiation of Th2 responses, that exaggerated Th1 responses contribute to autoimmunity and pulmonary pathology, and that Th1-like signal transduction pathway activation may also contribute to the pathogenesis of asthma (19).

The lung is unique among mucosal compartments in that it is constantly exposed to airborne antigens. As a consequence, the respiratory immune system must differentiate harmless antigens and potentially harmful infectious agents. This is done, in part, by a system of barriers that ensures that harmless antigens induce tolerance and do not elicit sensitization while potentially harmful exposures illicit sensitization and immune system activation. These tolerogenic pathways are altered in patients with asthma. As a result, asthmatics manifest a heightened ability to sensitize and to mount Th2 immune responses to antigens (for example, ragweed) that would not elicit similar responses in normal individuals. The details of the tolerogenic pathways in normal people and their defects in asthmatics are poorly understood. There is evidence that tolerance is mediated by special regulatory $\mathrm{T}$ cell populations ( $\mathrm{Tr}$ cells) that produce IL-10 or TGF- $\beta_{1}(2)$. Treatments that induce tolerance in asthmatics or people at risk for asthma are therefore of potential benefit in preventing and/or ameliorating asthmatic responses. Oral allergen immunotherapy and conventional allergen immunotherapy appear to work, in part, via the induction of tolerance. It is hoped that, over time, more effective toleranceinducing therapies will be developed.

\section{Major issues and future directions}

Disorders such as asthma are believed to be the result of a dysregulated mucosal immune system and pathologic $\mathrm{T}$ cell responses in genetically susceptible individuals (1). Over the next five to ten years we will need to define the relationships between the inflammatory response, the structural alterations noted in the remodeled asthmatic airway, and asthmatic symptomatology, physiologic dysregulation, and disease progression. These investigations will allow us to determine which inflammatory and structural changes contribute to disease pathogenesis (and thus need to be suppressed) and which are more reasonably thought of as aspects of an appropriate healing response in the injured airway (and thus should not be inhibited). It will also be essential to define the genetic alterations that contribute to asthmatic susceptibility, the genetic alterations responsible for the person-to-person variability in asthma presentation and severity, the processes regulating tolerance in the asthmatic and normal lung, and the mechanisms responsible for the lifelong nature of asthmatic inflammation. It is conceivable that, at some time in the future, DNA samples from patients with asthma or people at risk for asthma will be assessed for the presence or absence of polymorphisms of specific genes. Based on these assays, physicians would know whether the patient would develop asthma and, if so, what the natural history of the disease 
would be and what therapies the patient would optimally respond to. From this perspective, it is easy to see how our knowledge of asthma pathogenesis can have impressive ramifications for our practice of medicine. It is important to point out, however, that it has taken us over a century to get where we are. One is humbled by the appreciation that in $1892 \mathrm{Sir}$ William Osler, in The Principles and Practice of Medicine, said that

Asthma is a term which has been applied to various conditions associated with dyspnea... Of the numerous theories the following are most important:

1. It is due to spasm of bronchial muscles.

2. The attack is due to swelling of the bronchial mucous membrane.

3. In many cases it is a special form of inflammation of the smaller bronchioles.

Hopefully, it will not take us another century to go from our understanding of the inflammatory nature of the asthmatic diathesis to effective interventions that control and even prevent this common and debilitating disorder.

1. Neurath, M.F., Finotto, S., and Glimcher, L.H. 2002. The role of Th1/Th2 polarization in mucosal immunity. Nat. Med. 8:567-573.

2. Umetsu, D.T., McIntire, J.J., Akbari, O., Macaubas, C., and DeKruyff, R.H. 2002. Asthma: an epidemic of dysregulated immunity. Nat. Immunol. 3:715-720.

3. Finotto, S., et al. 2002. Development of spontaneous airway changes consistent with human asthma in mice lacking T-bet. Science. 295:336-338.

4. Ray, A., and Cohn, L. 1999. Th2 cells and GATA-3 in asthma: new insights into the regulation of airway inflammation. J. Clin. Invest. 104:1001-1006.

5. Tournoy, K.G., Kips, J.C., and Pauwels, R.A. 2002. Is Th1 the solution for
Th2 in asthma? Clin. Exp. Allergy. 32:17-29.

6. Zheng, T., et al. 2000. Inducible targeting of IL-13 to the adult lung causes matrix metalloproteinase- and cathepsin-dependent emphysema. J. Clin. Invest. 106:1081-1093.

7. Zhu, Z., Ma, B., Homer, R.J., Zheng, T., and Elias, J.A. 2001. Use of the tetracycline-controlled transcriptional silencer (tTS) to eliminate transgene leak in inducible overexpression transgenic mice. J. Biol. Chem. 276:25222-25299.

8. Zhu, Z., et al. 1999. Pulmonary expression of interleukin-13 causes inflammation, mucus hypersecretion, subepithelial fibrosis, physiologic abnormalities and eotaxin production. J. Clin. Invest. 103:779-788.

9. Temann, U.A., Ray, P., and Flavell, R.A. 2002. Pulmonary overexpression of IL-9 induces Th2 cytokine expression, leading to immune pathology. J. Clin. Invest. 109:29-39. doi:10.1172/JCI200213696.

10. Lanone, S., et al. 2002. Overlapping and enzyme-specific contributions of matrix metalloproteinases-9 and -12 in IL-13-induced inflammation and remodeling. J. Clin. Invest. 110:463-474. doi:10.1172/JCI200214136.

11. Zhu, Z., et al. 2002. IL-13-induced chemokine responses in the lung: role of CCR2 in the pathogenesis of IL-13-induced inflammation and remodeling. J. Immunol. 168:2953-2962.

12. Lee, C.G., et al. 2001. Interleukin-13 induces tissue fibrosis by selectively stimulating and activating TGF- $\beta_{1}$.J. Exp. Med. 194:809-821.

13. 1995. Global initiative for asthma: global strategy for asthma management and prevention. NHLBI/WHO workshop report. NIH, National Heart, Lung and Blood Institute. Bethesda, Maryland, USA. Publication number 95-3659.

14. Henderson, W.R., Jr., et al. 2002. A role for cysteinyl leukotrienes in airway remodeling in a mouse asthma model. Am. J. Respir. Crit. Care Med. 165:108-116.

15. Ito, K., et al. 2002. Expression and activity of histone deacetylases in human asthmatic airways. Am. J. Respir. Crit. Care Med. 166:392-396.

16. Ito, K., et al. 2002. A molecular mechanism of action of theophylline: induction of histone deacetylase activity to decrease inflammatory gene expression. Proc. Natl. Acad. Sci. USA. 99:8921-8926.

17. Milgrom, H., et al. 1999. Treatment of allergic asthma with monoclonal anti-IgE antibody. rhuMAb-E25 Study Group. N. Engl. J. Med. 341:1966-1973.

18. Donaldson, D.D., Elias, J.A., and Wills-Karp, M. 2001. IL-13 antagonism. In Progress in respiratory research: new drugs in asthma, allergy and COPD. Volume 31. T. Hansel and P. Barnes, editors. Karger Press. Basel, Switzerland. 260-263.

19. Sampath, D., Castro, M., Look, D.C., and Holtzman, M.J. 1999. Constitutive activation of an epithelial signal transducer and activator of transcription (STAT) pathway in asthma. J. Clin. Invest. 103:1353-1361. 